\title{
Análisis de reclamaciones judiciales contra obstetras y ginecólogos en España. Estudio específico de la vía penal (1987-2013).
}

García Ruiz, Nicolás, Santiago Sáez, Andrés, Albarrán Juan, María Elena, Labajo González, Elena y Perea Pérez, Bernardo.

Cita:

García Ruiz, Nicolás, Santiago Sáez, Andrés, Albarrán Juan, María Elena, Labajo González, Elena y Perea Pérez, Bernardo (2016). Análisis de reclamaciones judiciales contra obstetras y ginecólogos en España. Estudio específico de la vía penal (1987-2013). Revista Española de Medicina Legal, 42 (4), 136-141.

Dirección estable: https://www.aacademica.org/elenalabajogonzalez/41 ARK: https://n2t.net/ark:/13683/pcQr/gpq

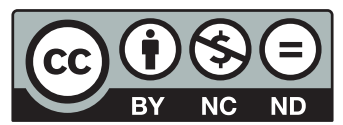




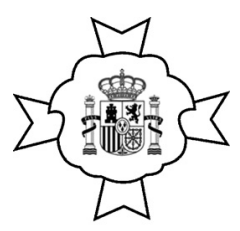

ASOCIACIÓN NACIONAL MÉDICOS FORENSES

\section{REVISTA ESPAÑOLA DE}

MEDICINA LEGAL

www.elsevier.es/mlegal

\title{
ORIGINAL
}

\section{Análisis de reclamaciones judiciales contra obstetras y ginecólogos en España. Estudio específico de la vía penal (1987-2013)}

\section{Nicolás García-Ruiz ${ }^{a}$, Andrés Santiago-Sáez ${ }^{\mathrm{b}, c, *}$, María Elena Albarrán-Juan ${ }^{\mathrm{b}}$, Elena Labajo-González ${ }^{\mathrm{b}}$ y Bernardo Perea-Pérez ${ }^{\mathrm{b}}$}

\author{
a Servicio de Obstetricia y Ginecología, Hospital El Escorial, Madrid, España \\ ${ }^{\mathrm{b}}$ Escuela de Medicina Legal y Forense, Universidad Complutense de Madrid, Madrid, España \\ c Servicio de Medicina Legal, Hospital Clínico San Carlos, Madrid, España
}

Recibido el 2 de marzo de 2016; aceptado el 28 de julio de 2016

\author{
PALABRAS CLAVE \\ Reclamaciones; \\ Vía penal; \\ Leyes penales; \\ Judicialización en \\ obstetricia
}

\begin{abstract}
Resumen
Objetivo: En España las reclamaciones judiciales contra ginecólogos resueltas por vía penal son escasas, menos del 3\%. Esta vía conlleva sanción económica y penas de cárcel y de inhabilitación. El objetivo es estudiar las sentencias resueltas por vía penal, por ser de consecuencias más graves y obtener un perfil del condenado.

Material y métodos: Análisis retrospectivo de las sentencias judiciales penales contra ginecólogos desde 1987 hasta 2013. Hemos utilizado tablas de distribución de Fisher, de normalidad y el t-Test para comparación de diferencias entre grupos y la prueba no paramétrica de MannWithney para comparación de muestras independientes. Para la caracterización de dos grandes grupos, el de «condenado» $\mathrm{y}$ «no condenado», realizamos estudio multivariante mediante análisis de correspondencias múltiples seguidas de análisis de conglomerados para agrupar.

Resultados: De 85 casos el 54,6\% de las reclamaciones judiciales procedían de actos médicos del sector público, aunque el mayor número de condenas, el 59,3\%, lo fue por demandas sobre hechos ocurridos en el ámbito de la sanidad privada. La probabilidad de condena en sucesos relacionados con el parto fue del 36,6\% (20 de 55) frente al 16,6\% (5 de 30) de episodios de otro origen. En el $69,2 \%$ de los casos de procedimientos penales por daño neurológico (encefalopatía neonatal) la sentencia fue condenatoria y con las indemnizaciones más elevadas. El tiempo medio trascurrido entre el incidente y la condena fue de 5,7 años. Con una condena previa, la posibilidad de confirmación en instancias superiores fue del $71,9 \%$. Una absolución se confirmó en el $96,2 \%$ de las ocasiones.
\end{abstract}

\footnotetext{
* Autor para correspondencia.

Correo electrónico: Andressebastian.santiago@salud.madrid.org (A. Santiago-Sáez).
} 


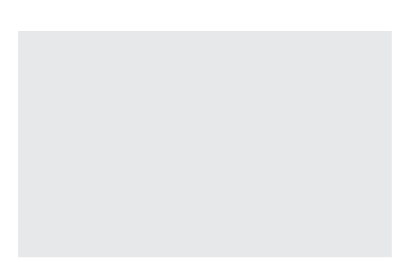

\section{KEYWORDS}

Malpractice claims;

Criminal court;

Criminal law;

Litigation in

obstetrics
Conclusiones: Aunque es elegida para iniciar el procedimiento pocas reclamaciones se resuelven en vía penal. La mayoría se deben a complicaciones durante el parto y en un tercio se condena. Lo más probable es que se confirme la sentencia previa cuando esta fue absolutoria.

(C) 2016 Asociación Nacional de Médicos Forenses. Publicado por Elsevier España, S.L.U. Todos los derechos reservados.

\section{Analysis of court claims filed against obstetricians and gynaecologists in spain. specific study of the criminal court system (1987-2013)}

\begin{abstract}
Introduction: Court claims against gynaecologists in the Spanish criminal court system are a rare occurrence (less than 3\%). These can lead to financial penalties, imprisonment and disqualification. Our aim is to study the court sentences issued in such cases, as these involve the most serious convictions, and to obtain a profile of those convicted.

Material and methods: A retrospective analysis of the sentences against gynaecologists during the period from 1987 to 2013. We used F-distribution tables, normal distribution tables and Student's t-test to compare differences between groups, and the Mann-Whitney nonparametric test for comparison of independent samples. In order to define two broad groups, those of "convicted" and "not convicted," we performed a multivariate study analysis followed by cluster analysis to determine similar sentences.

Results: Of the 85 cases, $56.4 \%$ of complaints came from procedures in the public sector, but the highest rate of convictions (59.3\%) was based on complaints originating in the private healthcare sector. The likelihood of conviction in events related to childbirth was $36.6 \%$ ( 20 out of 55 ) versus $16.6 \%$ (5 out of 30 ) in incidents of another origin. $69.2 \%$ of cases of criminal proceedings for permanent neurological damage (neonatal encephalopathy) concluded in a criminal conviction and had the highest financial compensation. The average time elapsed between the incident and the sentence was 5.7 years. With a prior conviction, the possibility of confirmation at higher levels was $71.9 \%$. An acquittal was confirmed in $96.2 \%$ of cases.

Conclusions: Of the court claims filed against gynaecologists based on their professional activity, very few are decided upon in the criminal courts. Most are due to complications during childbirth, and one-third ends with a conviction. A judgment of acquittal is most likely to be confirmed when this was the case with the previous one.

(C) 2016 Asociación Nacional de Médicos Forenses. Published by Elsevier España, S.L.U. All rights reserved.
\end{abstract}

\section{Introducción}

Entre las reclamaciones judiciales a médicos, algunas especialidades, como la obstetricia y ginecología ocupan un lugar destacado por el gran número que recibe, por el número de especialistas denunciados, y lo elevado de las indemnizaciones de las condenas.

En algunos países este incremento en las reclamaciones a ginecólogos se contempla como una «crisis» en esta especialidad ${ }^{1}$. En nuestro país existen pocas publicaciones científicas que permitan hacernos una idea del alcance del problema, un grupo en Cataluña, la correduría de seguros Uniteco y nuestro propio grupo ${ }^{2-4}$, son de los más activos en este aspecto. El grueso de las publicaciones, que consultamos y que utilizamos como referencia tiene su origen en el «escenario estadounidense ${ }^{5}$, muy alejado del nuestro en costumbres, sistema sanitario y sistema judicial y que reconocen como más costoso y menos efectivo ${ }^{6}$.

En España muchas reclamaciones se inician en la vía penal, porque es un procedimiento más económico y además en la vía penal también se depura la responsabilidad civil, es decir la cuantía de la indemnización económica ${ }^{7}$. Aquí, la obstetricia y ginecología es una de las especialidades médicas con más demandas judiciales y recibe el 15,53\% de todas las reclamaciones, solo por debajo de la traumatología y cirugía ortopédica $(15,69 \%)$. Globalmente considerada la especialidad y para sentencias de segunda o tercera instancia (en todas las vías), las condenatorias y absolutorias representan el mismo porcentaje, el 50\%. Específicamente, y de nuevo considerando todas las vías, es más probable la condena en el ámbito de la obstetricia, el $57,6 \%$, que en el de la ginecología, el $42,31 \%{ }^{4}$.

Aunque la vía penal es la vía elegida para el inicio de una reclamación judicial contra médicos, es en la que es más difícil que prospere la imputación. Es posible que los médicos desconozcan este hecho.

En todos los ámbitos se sabe que está aumentando el número de reclamaciones judiciales contra médicos, y este tiene más relevancia en alguna especialidad médica, como la obstetricia y ginecología, situada siempre entre los 
primeros lugares (3), y siendo la especialidad con mayor número de reclamaciones indemnizadas y con una tasa mayor de indemnizaciones extremas ${ }^{8}$.

Con frecuencia los datos proceden de las compañías de seguros que los utilizarán para hacer una valoración de riesgos y así ajustar las primas. Ello es así en nuestro país y también en otros con más tradición en el análisis de estos datos $^{8}$.

Existen muy pocos trabajos que se hayan acercado a este tema con un interés científico por lo que la información que recibe el profesional puede ser incompleta, pero se considera suficiente para provocar cambios asistenciales 9,10 como por ejemplo el incremento en el número de partos por cesárea $^{11,12}$.

También es cierto que esas «crisis de litigiosidad» como podríamos llamarlas, han colaborado, junto con el permanente interés de la ciencia médica por avanzar en la seguridad del paciente, al desarrollo de las guías clínicas ${ }^{13}$, y a la implantación de protocolos de actuación.

En nuestro estudio revisamos las sentencias de casos relacionados con la especialidad de ginecología y obstetricia desde 1987 a 2013, enjuiciados en la vía penal. Hasta donde sabemos, no existen referencias en la bibliografía médica sobre este aspecto. Consideramos que las condenas en esta vía pueden ser las que más influyan en los posibles cambios de actitud de los profesionales.

La jurisprudencia demuestra que es difícil que la imputación penal prospere ${ }^{14}$, pero como decimos, es esta la que más hace padecer al profesional implicado pues a la indemnización, hay que añadir penas de inhabilitación y de privación de libertad, junto con la difusión mediática.

Se opta en principio por la vía penal por sus ventajas económicas (casi gratis) y procesales y con la finalidad de evitar procedimientos más largos y farragosos ${ }^{14}$ y buscando la responsabilidad civil derivada de la penal. El recurso a la vía penal, en nuestra especialidad se está convirtiendo en un problema acuciante ${ }^{15}$, es por ello que el objetivo de nuestro trabajo es definir un «perfil del condenado» en vía penal en los años de estudio, intentando diferenciarlo de la vía civil, proclive al hecho jurídico de que no debe haber un perjuicio sin reparación ${ }^{15}$ y causa real del aumento de las sentencias sobre la responsabilidad civil contra médicos.

Necesitamos datos propios para comparar con los procedentes de otros países, principalmente Estados Unidos, cuyo sistema sanitario y judicial están muy alejados de los estándares europeos y más concretamente de los de nuestro país.

\section{Material y métodos}

Nos hemos nutrido fundamentalmente de la base de datos de sentencias de Westlaw Aranzadi, accediendo desde la Escuela de Medicina Legal de la Universidad Complutense de Madrid. Recogimos todas las sentencias por mala praxis médica emitidas contra ginecólogos, por actos médicos ocurridos después de 1987 y con la sentencia publicada antes de mayo de 2013, tramitados por la vía penal y que han alcanzado al menos la segunda instancia o superior, es decir Audiencia Provincial, Tribunal Superior de Justicia, o Tribunal Supremo. Esta base de datos contiene las sentencias íntegras. Las variables objeto de nuestro estudio fueron, el efecto adverso, el afectado por el daño reclamado, madre, feto o recién nacido, o paciente en el caso de la cirugía ginecológica, el tipo de cirugía y otras como el año del suceso y, el tiempo transcurrido entre este y la publicación de la sentencia definitiva, y el ámbito de la actividad médica, público o privado.

También hemos valorado si la sentencia definitiva ratificó o fue contraria a la de la instancia previa y en caso de condena la cantidad económica de la indemnización.

Para nuestro objeto fundamental, conocer el «perfil del condenado», hemos dividido el total de casos en dos grandes grupos, los relacionados con el parto y los relacionados con otros actos propios de la especialidad de obstetricia y ginecología, subclasificados a su vez por eventos adversos más frecuentes, según lo referido en publicaciones previas.

De las cantidades económicas resultantes de la indemnización, solo hemos hecho un análisis descriptivo.

Para el análisis estadístico hemos utilizado el software estadístico SPSS 19.0, las tablas de Fisher, de normalidad y el t-Test para la comparación de las diferencias entre grupos, y la prueba no paramétrica de Mann-Whitney para comparación de muestras independientes.

El análisis multivariante lo hemos realizado mediante el programa estadístico SPAD 7.0. y el análisis de correspondencias múltiples seguidas de un análisis de conglomerados para obtención de sentencias similares en cuanto a condena, parto y otros, y ámbito, y la caracterización de estos grupos homogéneos por estas y las demás variables, para la caracterización de dos grandes grupos, el de «condena» $\mathrm{y}$ «no condena».

\section{Resultados}

Del total de 85 sentencias, en la tabla 1 resumimos el origen de la denuncia, su relación o no con el parto, y el resultado. La mayor parte de las reclamaciones procedían de complicaciones surgidas en el parto, 55 y relacionadas con daño fetal, bien muerte del recién nacido, 25, o por daño neurológico

Tabla 1 Origen de la demanda, y resultado

\begin{tabular}{lcc}
\hline Acto médico & $\mathrm{N}(\%)$ & Condena N (\%) \\
\hline Parto & $55(64,7)$ & $20(36,6)$ \\
Déficit neurológico & $13(15,2)$ & $9(69,2)$ \\
Muerte fetal & $25(29,4)$ & $7(28,0)$ \\
Muerte materna & $4(4,7)$ & $2(50,0)$ \\
Distocia de hombros & $2(2,4)$ & $0(0,0)$ \\
Cuerpo extraño & $2(2,4)$ & $0(0,0)$ \\
Otros & $9(10,5)$ & $2(22,2)$ \\
No parto & $30(35,2)$ & $5(16,6)$ \\
Cáncer de mama & $6(20,0)$ & $1(16,6)$ \\
Complicaciones & $3(10,0)$ & $1(33,3)$ \\
$\quad$ laparoscopia & $4(13,3)$ & $1(25,0)$ \\
Complicaciones & & \\
$\quad$ histerectomía & $4(13,3)$ & $1(25,0)$ \\
Cuerpo extraño & $5(16,6)$ & $0(0.0)$ \\
Legrado/aborto & $2(6,6)$ & $0(0,0)$ \\
Cáncer de cérvix & $6(20)$ & $1(16,6)$ \\
Otros & $85(100)$ & $25(29,4)$ \\
Total & &
\end{tabular}


Tabla 2 Cuantías de las indemnizaciones

\begin{tabular}{|c|c|c|c|c|}
\hline Cuantía & Media & $\mathrm{DE}$ & $\operatorname{Min}$ & Máx \\
\hline \multicolumn{5}{|l|}{ Parto } \\
\hline Solicitada & $457.000,0$ & $367.046,3$ & 50.000 & 1.000 .000 \\
\hline Condena & $466.348,6$ & $403.995,5$ & 30.000 & 1.200 .000 \\
\hline \multicolumn{5}{|l|}{ No parto } \\
\hline Solicitada & $206.537,5$ & $155.714,8$ & 20.000 & 450.000 \\
\hline Condena & $124.200,0$ & $78.154,9$ & 6.000 & 200.000 \\
\hline$P$ & & & & 0,01 \\
\hline
\end{tabular}

Cantidades expresadas en euros. Reflejamos las cantidades crudas con actualización de la moneda, pero no se ha aplicado ningún factor corrector sobre las más antiguas.

residual, 13. En este último caso el $69,2 \%$ de las reclamaciones terminaron en condena y lo mismo ocurrió en el $28 \%$ de los casos de muerte fetal. Las denuncias no relacionadas con complicaciones en el parto y que se tramitaron por la vía penal fueron 30 en total. De estas, 6 (7\%) estaban relacionadas con retrasos diagnósticos de cáncer de mama, 3 $(3,5 \%)$, con complicaciones de la cirugía laparoscópica, y 4 $(4,7 \%)$ de histerectomía. De otra complicación de la cirugía el olvido de un cuerpo extraño encontramos 6 casos (7\%), 2 relacionados con el proceso de parto, y 4 con otros actos quirúrgicos y solo en un caso la sentencia fue condenatoria.

$\mathrm{El} 36,6 \%$ de las reclamaciones judiciales relacionadas con el parto terminaron en condena frente al $16,6 \%$ del resto.

Cuando la sentencia de origen fue condenatoria lo más probable es que también lo fuera la de instancia superior ( $72 \%$ de casos) pero fue más alta la posibilidad de que se confirmara una sentencia absolutoria $(96,2 \%)$.

La obstetricia requiere una estrecha colaboración entre el médico y la matrona, asumiendo esta una gran responsabilidad. Estudiamos por ello el profesional sobre el que recayó la reclamación. Únicamente sobre el médico, en 60 casos (70\%), sobre el médico y la matrona en $15(17 \%)$, y sobre la matrona exclusivamente en 8 ocasiones $(9,6 \%)$. En las dos últimas situaciones la posibilidad de condena fue ligeramente superior, del $37,5 \%$, frente al $28,3 \%$ de la primera.

De la sanidad pública procedían el $56,4 \%$ de las demandas, pero solo el $48 \%$ de las condenas, y del ámbito privado el 38,8 y el $59,3 \%$ respectivamente.

Respecto a la distribución temporal hemos encontrado una agrupación de casos entre los años 1995 y 2004, con un «pico» en el año 2002, y lo que parece un repunte en el año 2009 (fig. 1).

En las 25 condenas analizamos las cuantías solicitadas y las indemnizaciones concedidas. Las más elevadas lo fueron por denuncias sobre complicaciones en el parto, media de $457.000 €$, frente al resto, media de $124.200 €$ (tabla 2).

El tiempo transcurrido desde el año del suceso hasta la proclamación de la sentencia fue de 5,7 años de media, aunque con un rango muy amplio, de 2-15 años, por algunos pocos casos extremos, e independientemente de si la sentencia fue condenatoria o no.

El análisis multivariante (tabla 3) nos muestra que en la probabilidad de resultar condenado influyen fundamentalmente el que la sentencia previa fuera condenatoria, el daño neurológico permanente del recién nacido o

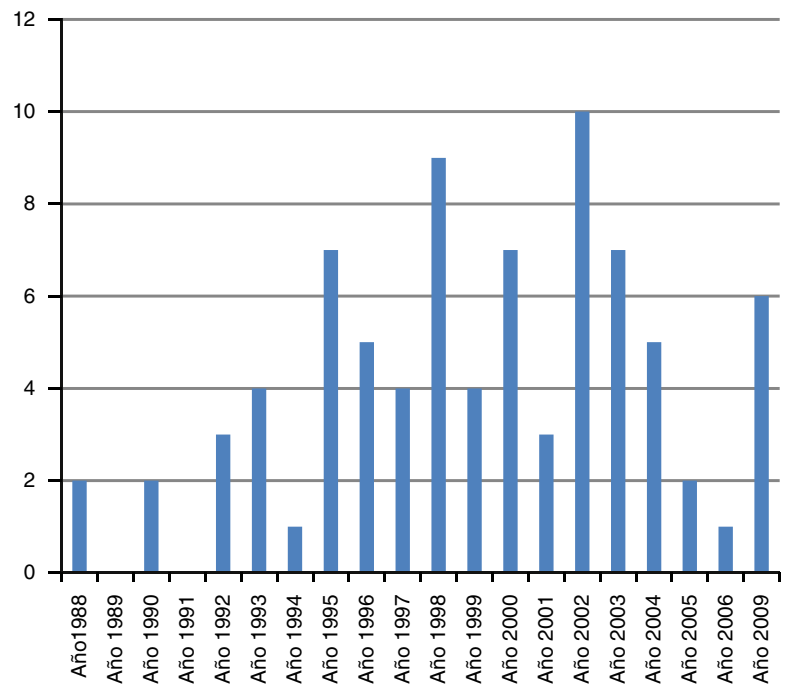

Figura 1 Jurisdicción penal, número de casos, distribución temporal.

Tabla 3 Análisis multivariante

\begin{tabular}{lll}
\hline & $t$-Test & $\mathrm{P}$ \\
\hline Confirmación de sentencia previa & 8,77 & 0,001 \\
Sentencia inicial condenatoria & 6,62 & 0,001 \\
Daño neurológico permanente & 1,96 & 0,025 \\
Ámbito privado & 2,12 & 0,017 \\
Parto & 1,83 & 0,034 \\
\hline
\end{tabular}

Variables con mayor relevancia estadística sobre el perfil del condenado.

relacionado con el parto en general como causa de la denuncia, y que el evento ocurriera en un ámbito privado.

\section{Discusión}

En nuestro estudio los eventos ocurridos en el parto, frente a los ocurridos fuera de él, los procedentes del ámbito privado, los relacionados con el daño neurológico permanente y aquellos en los que la sentencia previa fue condenatoria, fueron los factores que más estrechamente contribuyeron al «perfil del condenado» final. 
El análisis más específico de la vía penal, en segunda instancia o superior, pone de manifiesto que el porcentaje de sentencias condenatorias es del $29,4 \%$.

Más específicamente, en las mismas instancias, en la vía penal, el porcentaje de condenas en la obstetricia fue del $36,6 \%$ y para la ginecología del $16,6 \%$. Consideradas globalmente todas las vías, el porcentaje de condenas serían respectivamente 57,69 y $42,31 \%$.

Son raros los trabajos en nuestro entorno y en otros que se refieran a sentencias publicadas en la vía penal, lo que dificulta las comparaciones. Una publicación de una aseguradora francesa ${ }^{16}$ sí diferencia la «juridiction pénale et civile» y su sistema legal es comparable. En su caso, en causas relacionadas con la obstetricia sobre sentencias del año 2006, el porcentaje de condenas fue del $75 \%$ (de 8 demandas) en la vía penal y del $49 \%$ (de 39 demandas) en la civil.

En nuestro caso 85 sentencias resueltas en la vía penal en 25 años (desconocemos el número total de demandas), da una idea de que aunque esta es la de acceso más común, rara vez concluye en ella y que si los profesionales conocieran este dato, percibirían con menos angustia su «presencia» ante el juez.

Referente al «perfil del condenado» por la vía penal, la posibilidad de ser condenado es mayor cuando la reclamación judicial procede de actos médicos de la actividad privada, el $59,3 \%$ de condenas sobre 33 casos, que de la pública, el $48 \%$ de 48 , cuando está relacionada con eventos adversos ocurridos durante el parto, el $36,6 \%$ condenas de 55 casos, y cuando la causa está promovida por daño neurológico del recién nacido, el $69,9 \%$ condenas sobre 13 demandas. Este dato nos parece revelador porque ocurre a menudo que se cuestiona la relación entre la encefalopatía o asfixia neonatal con la mala praxis obstétrica, y si bien puede tener un origen multifactorial ${ }^{17}$, existe un número significativo de este tipo de eventos que pueden atribuirse o desarrollarse durante el parto ${ }^{18}$ y si se denuncian la posibilidad de condena en la vía penal es la más alta.

Las 20 condenas por hechos relacionados con el parto y 5 por otras causas en los 25 años que abarca nuestro estudio (1987-2013), no nos permiten un análisis sobre las cuantías económicas, ni evolutivo, ni comparativo, pero de los máximos y mínimos de las indemnizaciones podemos concluir con otros autores del entorno español ${ }^{19}$ que prácticamente todas las indemnizaciones catastróficas, definidas en España por ellos como aquellas superiores a $200.000 €$, lo son por hechos ocurridos en el parto, y si bien en su casuística estas suponen el 5,8\% del total, en la nuestra, en las condenas en la vía penal, son el $80 \%$ ( 20 de 25 ). Este y otros trabajos ${ }^{16,20}$, respaldan el hecho de que la obstetricia soporta las indemnizaciones más elevadas, y por tanto aquellas que superan el $1.000 .000 €$, lo cual también ocurre en nuestro caso. El rango en nuestra muestra se situó entre $6.000 €$ y $1.200 .000 €$, frente a los $200.775 €$ y $743.310,98 €$ del trabajo referido ${ }^{19}$. Estas diferencias se justifican por que el rango temporal, 25 vs. 6 años, no es comparable, tampoco el número de condenas, 25 frente a 550 , ni la vía judicial estudiada.

La cuantía económica y la gravedad de la lesión están relacionadas, pero pensamos que es más probable que las condenas en la vía penal influyan más en cambios en la actividad asistencial que las que solo son económicas, por elevadas que puedan ser. Además, solo el $5 \%$ de las reclamaciones judiciales en el ámbito jurídico-penal contra médicos llegan a la fase de enjuiciamiento ${ }^{21}$ y en esta especialidad, lo serán sobre todo por eventos adversos durante el parto y terminarán en absolución, más aún si lo fue la sentencia de procedencia. Este dato podría ayudar a revertir ese tipo de medicina «para evitar las demandas» o de «medicina defensiva», pero no es conocido, y esta ha sido otra de las intenciones de nuestro trabajo, dar a conocer lo difícil que resulta que una demanda que se inicia en la vía penal termine con una sentencia en ese ámbito.

Las diferencias encontradas entre el entorno público y privado en nuestro estudio, se deben interpretar teniendo en cuenta los importantes cambios ocurridos en los últimos años en los estándares asistenciales de la medicina privada, en determinadas zonas de España.

\section{Conclusiones}

Los casos de daño neurológico residual del recién nacido son los que tienen la probabilidad más alta de ser condenado y que la condena sea más dura. Es muy bajo el porcentaje de reclamaciones que alcanzan altas instancias en la vía penal, y aquí la probabilidad de resultar condenado es menor que en la vía civil e independiente de si se trata de un médico o de una matrona.

\section{Conflicto de intereses}

Los autores declaran no tener ningún conflicto de intereses.

\section{Bibliografía}

1. OB/GYN Rates and Risk of Malpractice: Considerations for the University of Connecticut Health Center Credentialing Committee"' (2009). UCHC Graduate School Masters Theses 2003-2010. Paper 158. [consultada 23 Jun 2013]. Disponible en: http://digitalcommons.uconn.edu/uchcgs_masters/158

2. Gómez-Duran EL, Lailla-Vicens JA, Benet-Travé J, ArimanyManso J. Analysis of obstetrics and gynecology profesional liability claims in Catalonia, Spain (1986-2010). J Forensic Leg Med. 2009;16:301-6.

3. Las reclamaciones a médicos se duplican (página en internet). Madrid: Uniteco Profesional Correduría de Seguros. [consultado 18 Nov 2014]. Disponible en: http://www.unitecoprofesional. es/blog/wp-content/uploads/2012/05/230512_Lasreclamaciones-a-m\%C3\%A9dicos-se-duplican-VOK.pdf

4. Pérez Pérez B, Santiago Saez A, Labajo González ME, Albarrán Juan MI, Sánchez Sánchez JA. Análisis de las consecuencias médico-legales de las reclamaciones judiciales de pacientes. Estudio comparativo de los casos de traumatología, ginecología y obstetricia, anestesia y reanimación, y odontoestomaología. Trauma Fund Mafre. 2009;20:264-8.

5. Arimany-Manso J, Gómez-Durán EL, Aubia-Marimon J. Las reclamaciones sobre responsabilidad profesional por especialidades en un escenario diferente al estadounidense. Gac Sant. 2013;27:89-93.

6. aos.org (página en internet). Worldwide trends in medical liability. Rosemont, Illinois: American Academy of Orthopaedic Surgeons; [consultado 30 Feb 2014]. Disponible en: http://www. aaos.org/news/aaosnow/sep11/managing4.asp

7. Llamas Pombo E. La responsabilidad civil del médico. Aspectos tradicionales y modernos. Madrid: Trivium.1988. 
8. Jena $A B$,Seabury S, Lakdawalla $D$, Chandra A. Malpractice risk according to physician specialty. N Engl J Med 2011; 365:629636.

9. Committee on Professional Liability. Coping with the stress of malpractice litigation: number 150 - December 1994. Int J Gynaecl Obstet. 1995;49:83-4.

10. Xu X, Siefert KA, Jacobson PD, Lori JR, Ransom SB. The effects of medical liability on obstetric care supply in Michigan. Am J Obstet Gynecol. 2008;198:1-9.

11. Yang YT, Mello MM, Subramanian SV, Studdert DM. Relationship between malpractice litigation pressure and rates of caesarean section and vaginal birth after caesarean section. Obstet Gynecol. 1991;78:1050-4.

12. Schifrin BS, Cohen WR. The effect of malpractice claims on the use of caesarean section. Best Pract Res Clin Obstet Gynaecol. 2013;27:269-83.

13. Mello MM, Studdert DM, Brennan TA. The new medical malpractice crisis. N Engl J Med. 2003;348:2281-4.

14. Sánchez García MM. La responsabilidad médica y los derechos del paciente. En: Sociedad Española de Obstetricia y Ginecología, editor. Problemas Jurídicos en Obstetricia y Ginecología. Madrid; 2013. p. 5-23.

15. De Lorenzo O. La responsabilidad penal de los especialistas en obstetricia y ginecología. En: Sociedad Española de Obstetricia y Ginecología, editor. Aspectos jurídicos en Obstetricia y Ginecología. Madrid; 2008. p. 251-87.
16. Le risque des professions de santé en 2006 (página en internet). Decisions de justice. Rapport sur les décisions de justice rendues en 2006 (médecins sociétaires du Sou Médical - Groupe MACSF). Responsabilité, Revue de formation sur le risque médical. [consultada 7 Feb 2014]. Disponible en: http://www.cfef. org/archives/bricabrac/macsf2006.pdf

17. Johnson SL, Blair E, Stanley FJ. Obstetric malpractice litigation and cerebral palsy in term infants. J Forensic LegMed. 2011;8:97-100.

18. Jonsson M, Agren J, Nordén-Lindeberg S, Ohlin A, Hanson U. Neonatal encephalopathy and the association to asphyxia in labor. Am J Obstet Gynecol. 2014;211:667e1-8.

19. Arimany-Manso J, Gómez-Durán EL, Barbería-Marcalain E, Benet-Travé J, Martin-Fumadó C. Catastrophic medical malpracticepayouts in Spain. J Healthc Qual. 2015 [Publicación electrónica].

20. Bixenstine PJ, Shore AD, Mehtsun WT, Ibrahim AM, Freischlag JA, Makary MA. Catastrophic medical malpractice payouts in the United States. J Healthc Qual. 2014;36:43-53.

21. derecho.publicacionmedica.com (página en internet). Asociación Española de Derecho Sanitario; [actualizado Jun 2016, consultada 3 May 2015]. Disponible en: http://derecho. publicacionmedica.com/noticia/solo-el-5-de-las-demandaspenales-contra-los-medicos-llegan-a-la-fase-de-enjuiciamiento 